\title{
Anomalous temperature dependence of the current in a metal-oxide-polymer resistive switching diode
}

\author{
Henrique L. Gomes ${ }^{1 \text { a) }}$, Paulo R. F. Rocha ${ }^{1}$, Asal Kiazadeh ${ }^{1}$ \\ Dago M. De Leeuw ${ }^{2}$ and Stefan C. J. Meskers ${ }^{3}$
}

${ }^{1}$ Center of Electronics Optoelectronics and Telecommunications (CEOT) Universidade do Algarve, Campus de Gambelas, 8005-139 Faro, Portugal

${ }^{2}$ Philips Research Laboratories, Professor Holstlaan 4, 5656 AA Eindhoven The Netherlands

${ }^{3}$ Molecular Materials and Nanosystems, Eindhoven University of Technology, P.O. Box 513, 5600 MB Eindhoven, The Netherlands

\begin{abstract}
Metal-oxide polymer diodes exhibit non-volatile resistive switching. The currentvoltage characteristics have been studied as function of temperature. The lowconductance state follows a thermally activated behavior. The high-conductance state shows a multistep-like behavior and below $300 \mathrm{~K}$ an enormous positive temperature coefficient. This anomalous behavior contradicts the widely held view that switching is due to filaments that are formed reversibly by the diffusion of metal atoms. Instead, these findings together with small-signal impedance measurements indicate that creation and annihilation of filaments is controlled by filling of shallow traps localized in the oxide or at the oxide/polymer interface.
\end{abstract}

Keywords: resistive switching, non-volatile memory, bistability, electrical properties, traps.

PACS: 73.20.Hb, 73.40.Qv 73.61.Ng

a) Electronic mail: $\underline{\text { hgomes@ualg.pt }}$ 


\section{Introduction}

Resistive switching has been observed in metal-insulator metal (MIM) diodes for a wide variety of insulator such as oxides, nanoparticles, organic or inorganic semiconductors, and currently attracts attention for application in future non-volatile memories [1]. We have recently shown that functional memories can be obtained by inserting a thin oxide layer into a polymer diode [2]. A yield of switching diodes of almost unity has been reported. Switching is a generic property of metal oxides [3-6], the polymer only acts as a current limiting series resistance [7]. The memory is formed by applying a high bias pulse to the pristine diode. After this so-called forming process, attributable to soft breakdown of the oxide [2], the memory can be switched reversibly between a high conductance ON-state and a low conductance OFF-state. Thermal imaging has shown that the conduction in the ON-state is filamentary in nature [8]. At present, the nature and formation of these filaments is not clear. Several models for the switching have been proposed such as metal filaments that rupture locally by Joule heating, $[9,10]$ or trap controlled tunneling between metal islands [11].

In order to distinguish between these mechanisms, we focus on the temperature dependence of the current for an oxide-polymer switching device. The anomalous positive temperature coefficient (PTC) that we observe below $300 \mathrm{~K}$, shows that more filaments become active when the device is cooled down. This observation contradicts explanations based on atomic diffusion. We argue that filaments switch on and off by filling and emptying of shallow trap states located in the oxide layer or at the polymer/oxide interface. This hypothesis is supported by small signal impedance measurements. 


\section{Experimental}

Pristine Metal-Insulator-Metal (MIM) diodes consisted of an Al bottom electrode, a sputtered layer of $\mathrm{Al}_{2} \mathrm{O}_{3}(10 \mathrm{~nm})$, a spirofluorene polymer $(80 \mathrm{~nm})$, and a $\mathrm{Ba} / \mathrm{Al}(5 \mathrm{~nm} / 100 \mathrm{~nm})$ top electrode (see figure 1). The diodes with an active area of 9 $\mathrm{mm}^{2}$ and $1 \mathrm{~mm}^{2}$ were encapsulated to exclude atmospheric contamination. The current-voltage (I-V) curves were obtained using a Keithley 487 picoammeter voltage source and capacitance-frequency and capacitance-voltage $(\mathrm{C}-\mathrm{V})$ curves were obtained using an Agilent 4192 impedance analyzer. Temperature dependent measurements were carried out in a liquid helium cryostat (Advanced Research Systems, ARS - HC2). Positive bias voltage refers to the bottom electrode being poled positive with respect to the top electrode.

\section{Results and discussion}

The pristine devices were turned into programmable resistive switching memories by applying a $12 \mathrm{~V}$ voltage ramp as described previously [2, 12]. After forming, the devices exhibit the usual bistable $J-V$ characteristics and a negative differential resistance (NDR) in the ON state, see figure 2. At room temperature, the memory can be switched between OFF and ON-state by applying voltage pulses with amplitudes corresponding to the top and bottom of the NDR [13], here about 3-4 V and 6-8 V respectively.

A stable OFF-state is induced by applying high bias voltage for a long time, in the order of minutes. The time necessary to induce a switching to the ON-state is now much longer than the time necessary to record a full $J$ - $V$ curve. In agreement with literature [14], the electrical current in the programmed OFF-state follows a thermally 
activated behavior (see figure 3). The Arrhenius plot of the current measured at $1 \mathrm{~V}$ is shown in the inset; yielding activation energy of $66 \mathrm{meV}$ for the temperature range of 280-200 K. Below $200 \mathrm{~K}$, the current is not thermally activated.

Interestingly, the $J-V$ characteristics for the ON-state show a large increase in the magitude of the current upon lowering the temperature of the diode. This behaviour is illustrated in figure 4. The increase in current is more pronounced at higher bias voltage, in the voltage range below the sharp onset of the NDR. The lower the temperature the higher is the swicthing-off volatge. The physical mechanims behind this shfit in voltage is under study.

To further explore this unusual temperature depedence, the diode was programmed into the $\mathrm{ON}$-state at room temperature and then cooled down while applying a continous bias voltage $(2 \mathrm{~V})$. The magnitude of the current more than doubles in a temperature range of 150 degrees, see figure 5. This corresponds to a positive temperature coefficient (PTC) of the electrical resistivity, $\alpha \approx 0.01 \mathrm{~K}^{-1}$, an anomalously large value when compared with typical values for metals $\left(\alpha=0.0039 \mathrm{~K}^{-1}\right.$ for $\mathrm{Cu}$ ). Furthermore, in contrast to the smooth decrease of the resistivity in metals, the resistivity of the diodes lowers in a step-like fashion upon cooling.

The step-like nature of the rise in current, strongly suggest that additional conducting filaments become active at low temperature. In order to check for selfheating effects during the experiments, we also carried out in other sample cooling runs where the applied bias was temporarily removed for intervals of times ranging from a few seconds to minutes, allowing for thermal equilibration. During these times the current across the sample becomes zero. In these runs, a similar increase in conduction was observed as shown in figure 6. A small section of the curve is magnified to shown that the rise in current is trough small discrete events (see inset). Furthermore, the PTC 
of the current is independent of heating speed and direction of temperature ramping. Thus self-heating effects can be disregarded. Small PTC effects were reported by others [14-16] and attributed to metallic filaments.

The abnormally large PTC cannot be explained by a metallic type of conduction. The large and stepwise increase in current can be rationalized by the temperature dependence of trap occupancy. Here we assume that shallow trap sites around the Fermi level could control the activity of filaments. At room temperature they are depopulated by thermal emission. However, upon cooling the diode, the shallow traps will be gradually filled and activate filaments.

In order to test the reported hypothesis that these trap sites are located in the oxide, [10] we performed small-signal impedance measurements. Figure 7 shows the temperature dependence of the capacitance $(C)$ and loss $(G / \omega)$ where $G$ is the conductance and $\omega$ is the angular frequency. The low-frequency capacitance remains constant. At low frequencies the loss is proportional to $1 / \omega$, which implies a constant DC conductance. As shown in figure 7, upon decreasing the temperature, the low frequency conductance increases. This is in agreement with the PTC observed for the DC conductance as shown in figure 5. The temperature dependence of the loss recorded with a signal test frequency of $300 \mathrm{~Hz}$ is shown in figure 8 . Similar to the DC measurements, the loss shows a discontinuous, step-like increase upon cooling. In previous work [7], we have shown that the frequency dependent response of the diode can be modeled as a double RC circuit represented in the inset of figure 7 . At low frequency the response is determined by the properties of the oxide layer, while at higher frequencies the polymer layer dominates. This equivalent circuit was used to fit the loss curves recorded at different temperatures (shown in figure 9). Since the measured capacitance does not change significantly with temperature, changes are 
mostly resistive and only located in the oxide layer (represented as $\mathrm{R}_{\mathrm{OX}}$ ). The dependence of $\mathrm{R}_{\mathrm{OX}}$ with temperature as estimated from the equivalent circuit is shown in the inset of figure 9. A decrease in $\mathrm{R}_{\mathrm{OX}}$ from $160 \mathrm{k} \quad$ (at $300 \mathrm{~K}$ ) to $6.6 \mathrm{k} \quad$ (at $124 \mathrm{~K}$ ) is required to provide a good fit to the data. The increase of the loss at low frequencies indicates that the oxide conductance increases upon cooling. Therefore, the trap sites active in switching are located in the oxide or at the oxide polymer interface.

For these memory devices, the presence of shallow traps cannot be detected by smallsignal impedance measurements because the large DC current across the sample mask the contribution of traps to the low-frequency capacitance.

Concerning to the chemical nature of the trap sites, we mention the possibility of a water-related defect. This defect is known to contribute to temperature dependent changes in electrical properties at temperatures around $200 \mathrm{~K}$, related to a phase transition [17-19]. The fact that both the dielectric loss and DC current do not change significantly near $200 \mathrm{~K}$ suggests that the water-related traps do not contribute to the PTC effect. Furthermore, the PTC effect is still observed for temperatures as low as 130 $\mathrm{K}$ which is well below $200 \mathrm{~K}$, temperature where the water related defect becomes a trap for charge carriers. This strongly suggests that although water-related traps maybe be present, they are not responsible for the PTC effect reported here.

In order to get insight into the trap characteristics, thermally stimulated currents were also attempted. However, switching diodes shown relatively noisy currents, which hinder the observation of discernible structure in thermally stimulated currents.

\section{Conclusions}

We note that the commonly reported view that filaments are created by the diffusion of metallic species from the electrode into the bulk region cannot explain the abnormal 
positive temperature coefficient. Instead we argue that in the forming process conducting paths are preformed. Here diffusion of metal atoms can be involved, as indicated by a number of experiments $[5,16,20]$. However, this does not imply that every time the memory switches, a filament is formed or ruptured by diffusion of atoms. Instead, our findings support that preformed paths exist and that these are turned on and off by filling and emptying of trap states, acting as micro-switches. Equivalent circuit modeling shows that these micro-switches are physically located in or near the oxide layer.

\section{Acknowledgements}

We gratefully acknowledge the financial support received from the Dutch Polymer Institute (DPI), project 703, from Fundação para Ciência e Tecnologia (FCT) through the research Unit, Center of Electronics Optoelectronics and Telecommunications (CEOT), REEQ/601/EEI/2005 and the POCI 2010, FEDER. 


\section{References}

[1] Scott J C and Bozano L D 2007 Adv. Mater. 191452.

[2] Verbakel F, Meskers S C J, Janssen R A J, Gomes H L, Cölle M. Büchel M, and de Leeuw D M 2007 Apply. Phys. Lett. 91192103.

[3] Beck A, Bednorz J G, Gerber C, Rossel C, Widmer D 2000 Appl. Phys. Lett. 77139.

[4] Waser R, Aono M 2007 Nature Mater. 6833.

[5] Karthäuser S, Lüssem B, Weides M, Alba M, Besmehn A, Oligschlaeger R, Waser R 2006 J. Appl. Phys. 100094504.

[6 ] Oyamada T, Tanaka H, Matsushige K, Sasabe H, Adachi C 2003 Appl. Phys. Lett. 831252.

[7] Gomes H L, Benvenho A R V, de Leeuw D M, Cölle M, Stallinga P, Verbakel F and Taylor D M 2008 Organic Electron. 9119.

[8] Cölle M, Büchel M, de Leeuw D M 2006 Organic Electronics 7305.

[9 ] Dearnaley G, Morgan D V, Stoneham A M 1970 J. Non-Crys Solids 4593.

[10] Dearnaley G, Stoneham A M, Morgan D V 1970 Rep. Prog. Phys. 331129.

[11] Thurstans R E, Oxley D P 2002 J. Phys. D. 35802.

[12] Simmons J G and Verderber R R 1967 Proc. R. Soc. London, Ser. A. 30177.

[13] Bozano L D, Kean B W, Deline V R, Salem J R and Scott J C 2004 Appl. Phys. Lett. 84607.

[14] Tondelier D, Lmimouni K, Vuillame D, Fery C and Haas G 2004 Apply. Phys. Lett. 855763.

[15] Chen L, Xia Y, Liang X, Yin K, Yin J, Liu Z, Chen Y 2007 Appl. Phys. Lett. 91 073511. 
[16] Joo W J, Choi T L, Lee K H and Chung Y 2007 J. Phys. Chem. B. 1117756.

[17] Gomes H L, Stallinga P, Cölle M and de Leeuw D M, and Biscarini F 2006 Appl. Phys. Lett. 88082101.

[18] Debenedetti P G 2003 J. Phys.: Condens. Matter. 15 R1669.

[19] Mishima O and Stanley H E 1988 Nature (London) 392164.

[20] Ma L, Xu Q, and Yang Y 2004 Appl. Phys. Lett. 844908. 


\section{Figure captions}

Fig. 1. (a) Schematic diagram showing the physical structure of the memory device, (b) photograph of an encapsulated device containing a number of diodes.

Fig. 2. $J-V$ characteristics of the device in the OFF and in the ON-state.

Fig. 3. Typical $J-V$ characteristics for the OFF-state. The inset shows the Arrhenius plot of the current measured at the voltage of $1 \mathrm{~V}$.

Fig. 4. Temperature dependence of the $J-V$ curve of a diode programmed into the ONstate.

Fig. 5. The temperature dependence of the current in the high-conductance ON-state monitored at $2 \mathrm{~V}$. The cooling speed is $1 \mathrm{~K} / \mathrm{min}$.

Fig. 6. The temperature dependence of the current in the high-conductance ON-state monitored at $1 \mathrm{~V}$ in a similar sample to the one shown in figure 5. In this cooling run the bias was temporarily removed for time intervals ranging from a few seconds to minutes, allowing for thermal equilibration. The cooling speed is $1 \mathrm{~K} / \mathrm{min}$. The inset shows the discrete nature of the current variation with temperature.

Fig. 7. Frequency dependence of the capacitance $(C)$ and the loss $(G / \omega)$ in the ON state. The inset shows the corresponding equivalent circuit which describes the device as a double-layer structure. 
Fig. 8. Low-frequency loss $(G / \omega)$, of the diode as function of temperature.

Measurements were carried out at with a test frequency of $300 \mathrm{~Hz}$. The solid line is a guide to eye.

Fig. 9. Experimental measured loss (points) and theoretical fits (continuous lines) using the equivalent circuit of figure 7 . The changes are resistive and located in the oxide layer of the sample; only changes in $\mathrm{R}_{\mathrm{OX}}$ are necessary to account for the changes in the loss with temperature. All other circuit parameters were kept constant. The inset shows the values of $\mathrm{R}_{\mathrm{OX}}$ used to fit the loss at a particular temperature. 

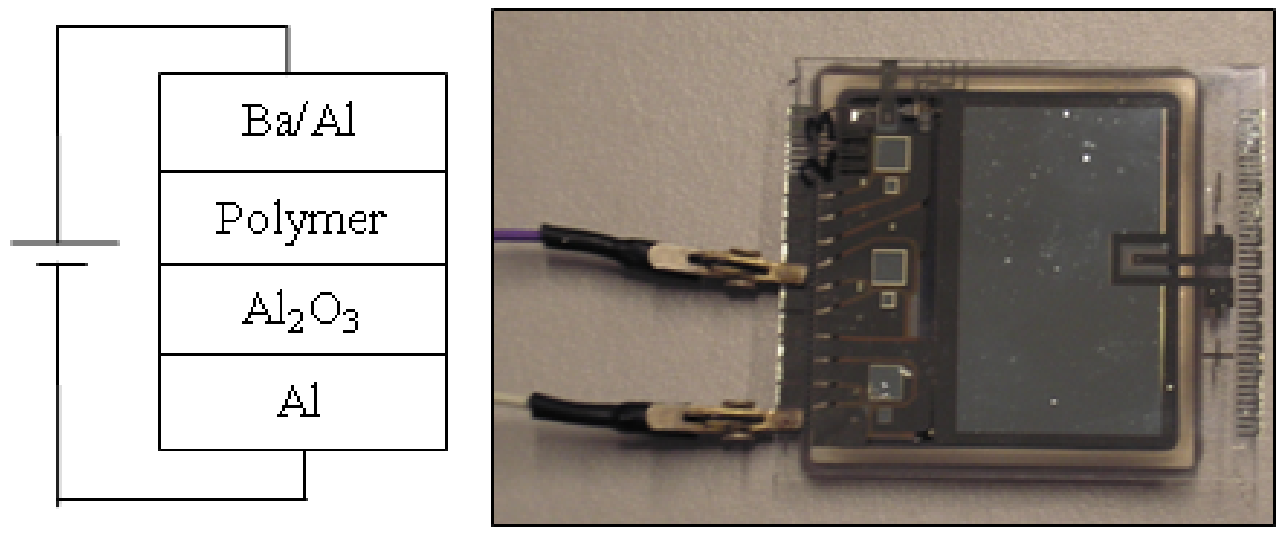

Figure 1

Figure 1 (Fig_1.tif) 


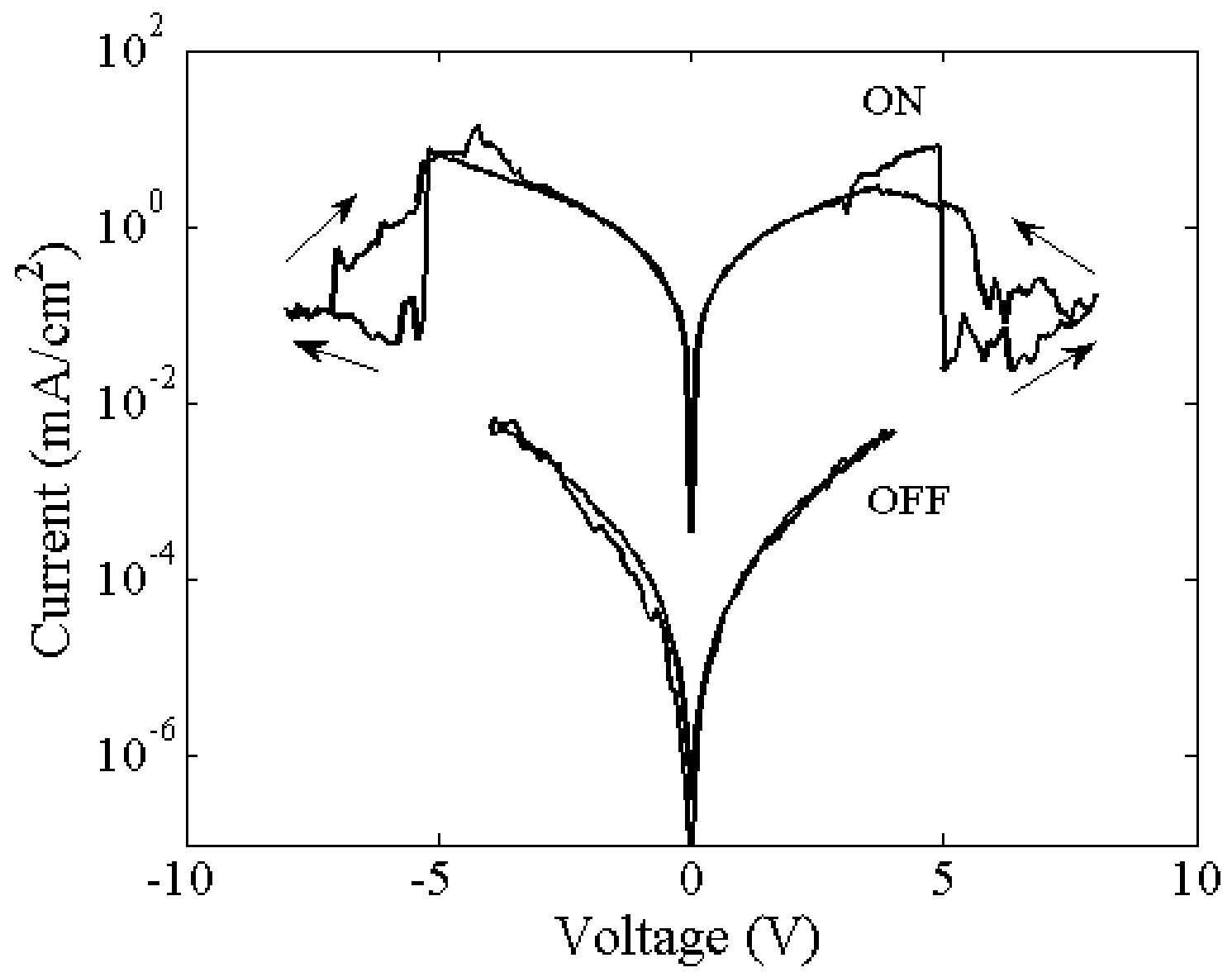

Figure 2

Figure 2 (Fig_2.tif) 


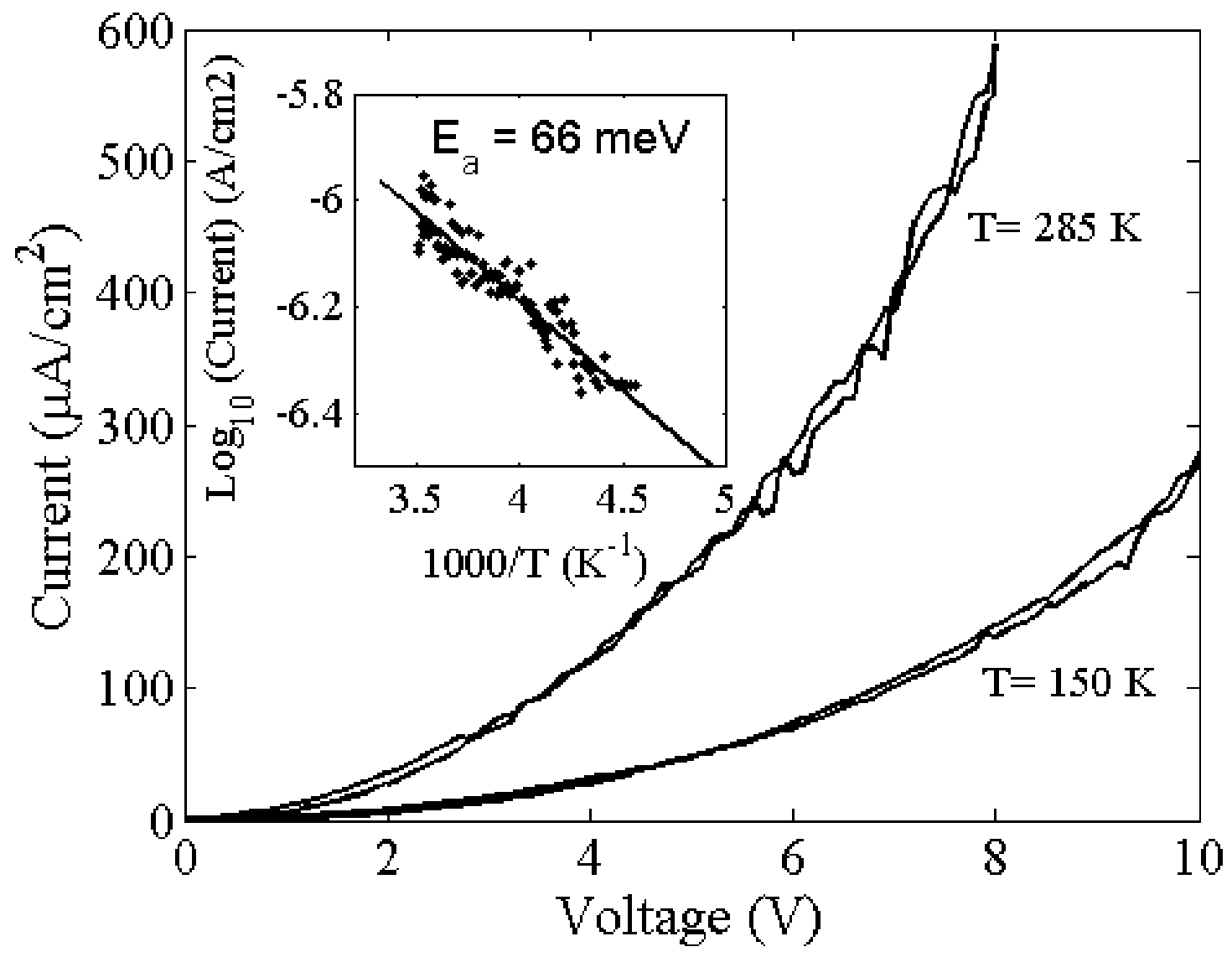

Figure 3

Figure 3 (Fig_3.tif) 


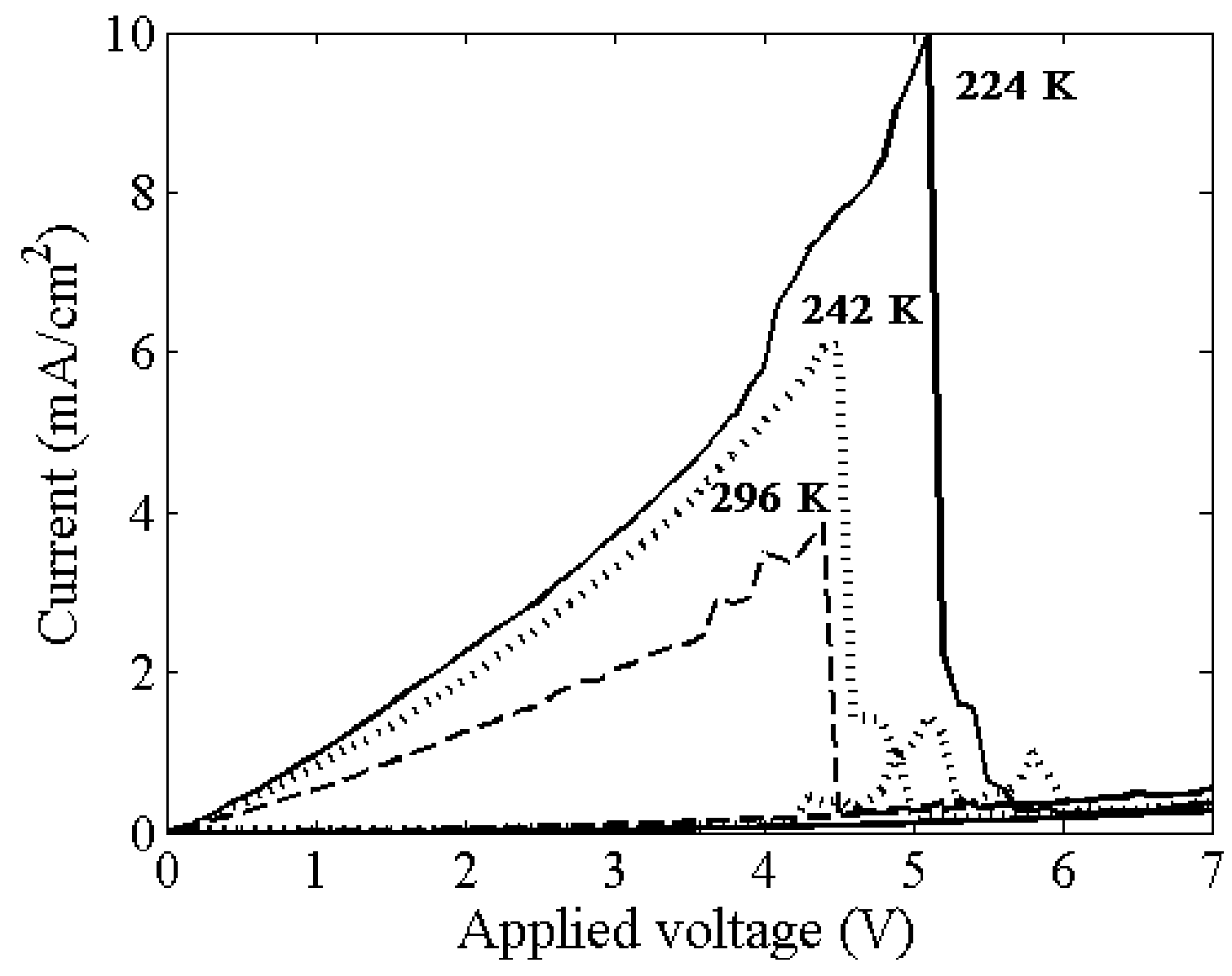

Figure 4

Figure 4 (Fig_4.tif) 


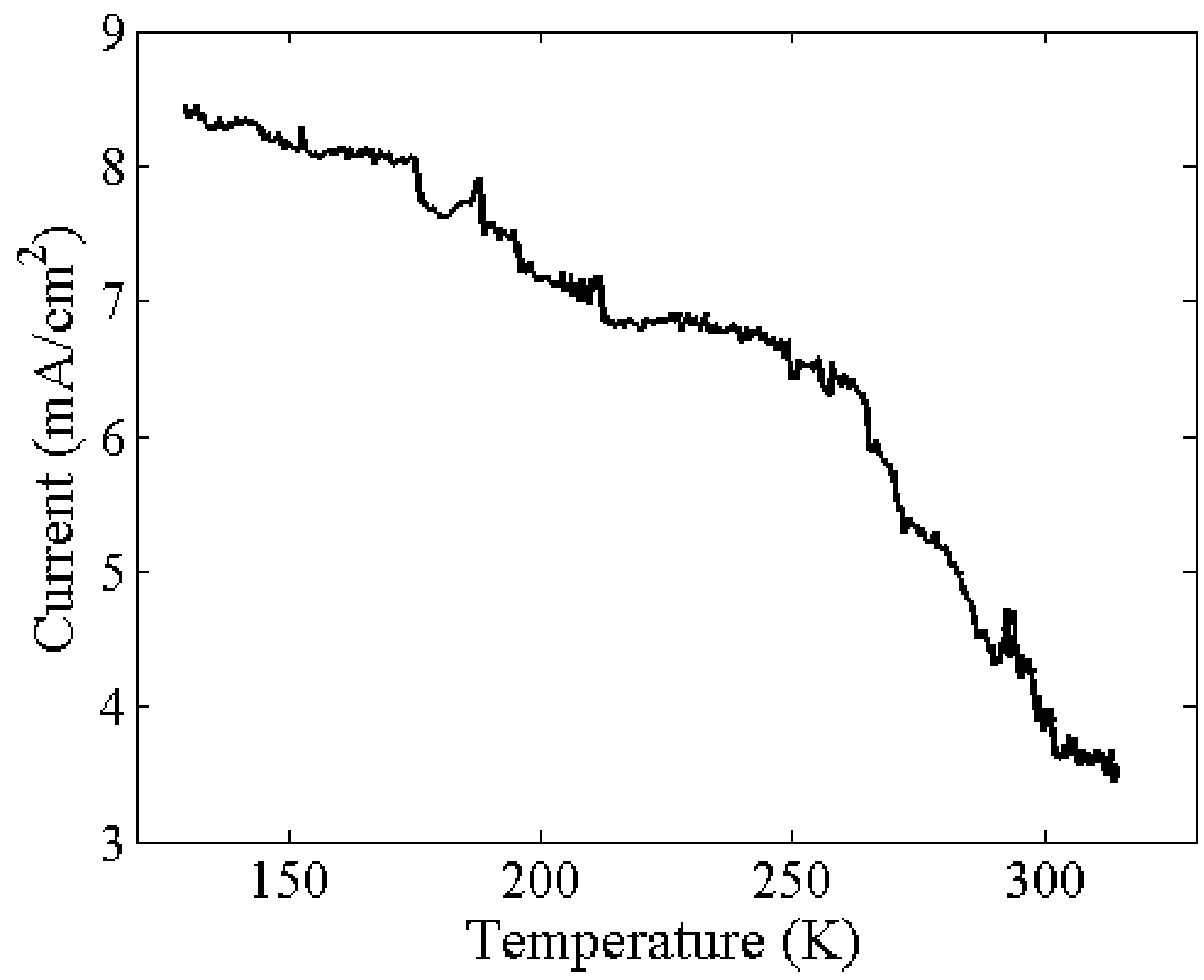

Figure 5 (Fig_5.tif) 


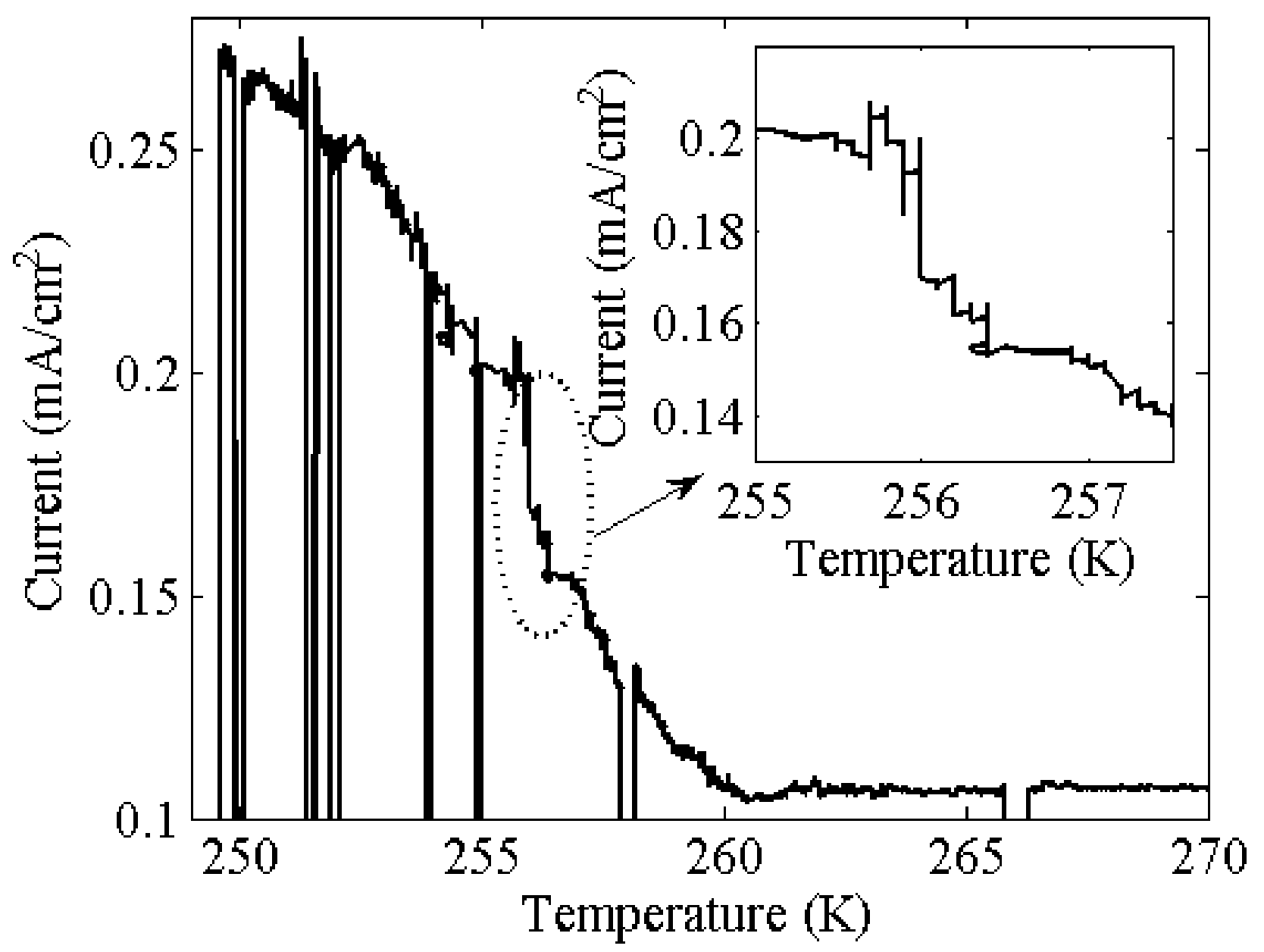

Figure 6 (Fig_6.tif) 


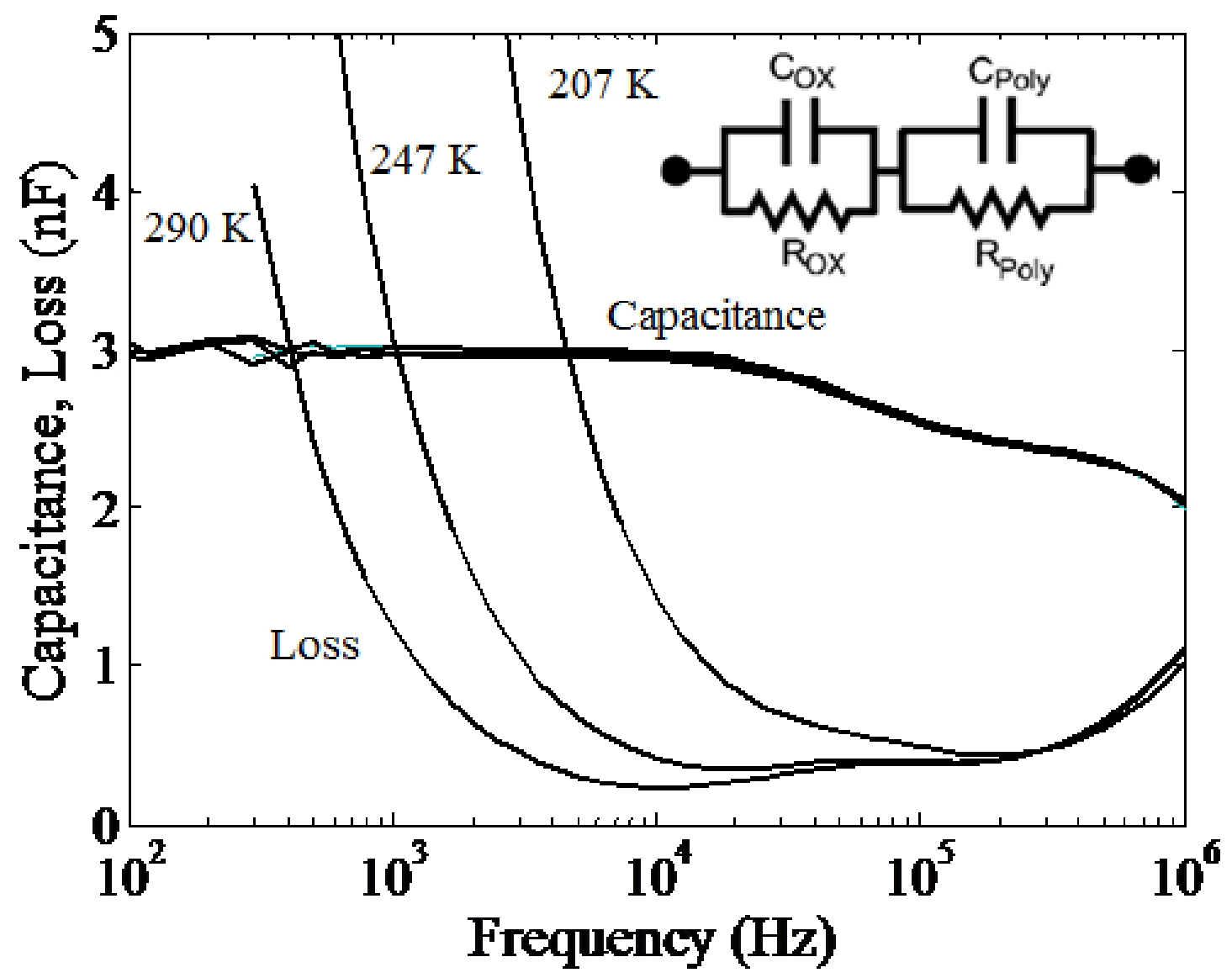

Figure 6

Figure 7 (Fig_7.tif) 


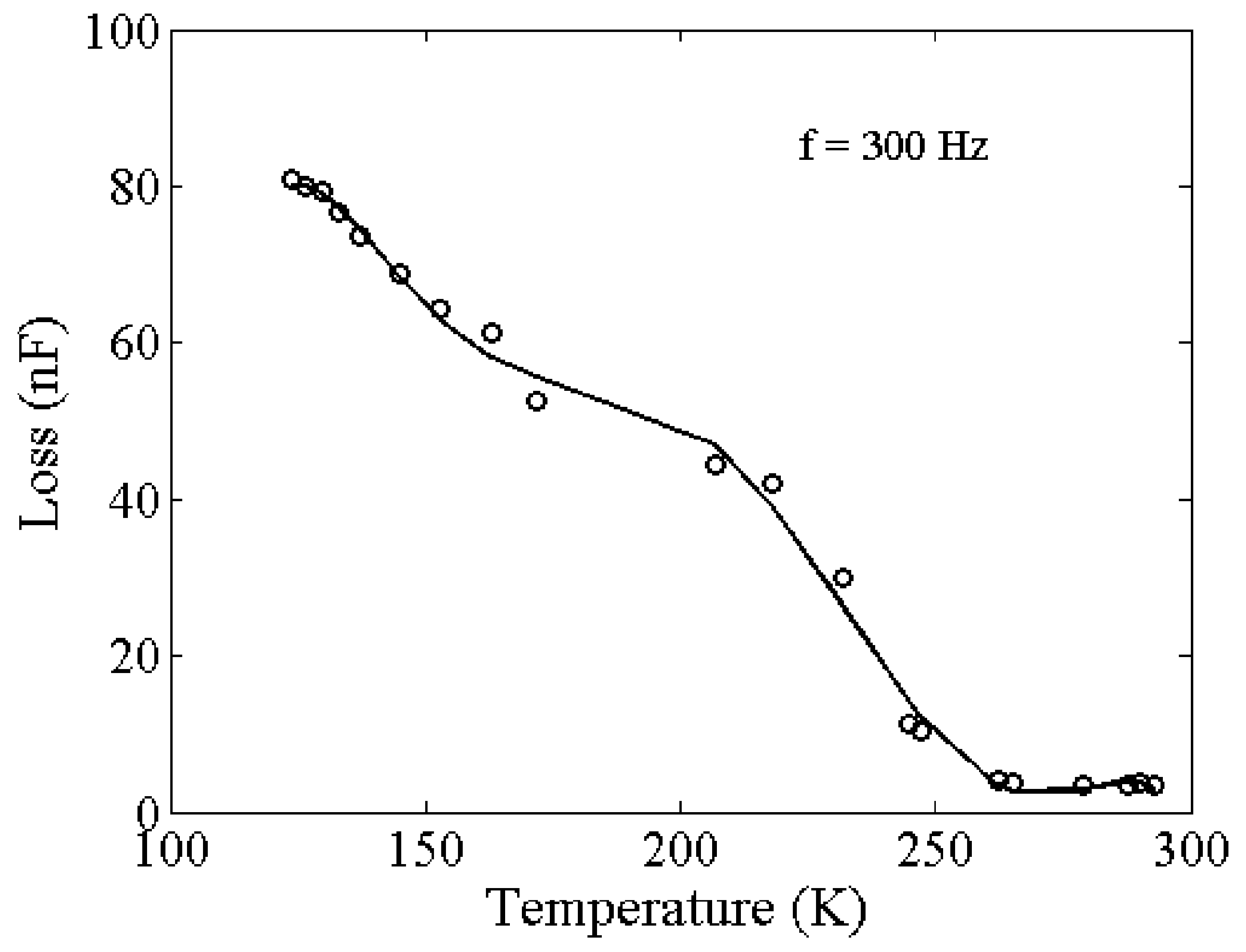

Figure 7

Figure 8 (Fig_8.tif) 


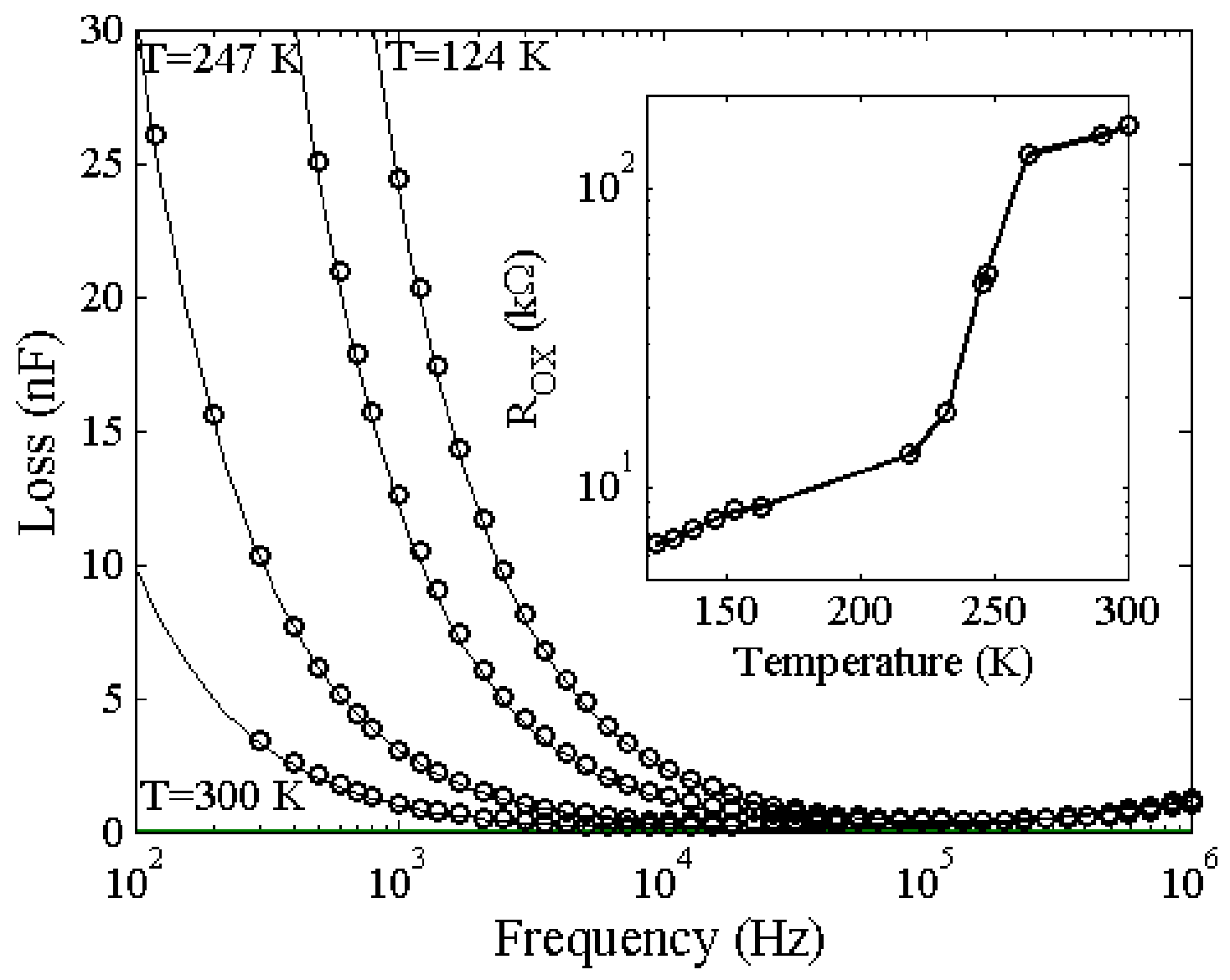

Figure 8

Figure 9 (Fig_9.tif) 